\title{
Effective Scalar Field Theory and Reduction of Couplings.
}

\author{
Mario Atance周 and José Luis Cortés门 \\ Departamento de Física Teórica, \\ Universidad de Zaragoza, 50009 Zaragoza, Spain.
}

\begin{abstract}
A general discussion of the renormalization of the quantum theory of a scalar field as an effective field theory is presented. The renormalization group equations in a mass-independent renormalization scheme allow us to identify the possibility to go beyond the renormalizable $\phi^{4}$ theory without losing its predictive power. It is shown that there is a minimal extension with just one additional free parameter (the mass scale of the effective theory expansion) and some of its properties are discussed.
\end{abstract}

PACS number(s): 11.10.Hi

Keywords: Effective Lagrangian, Renormalization.

*E-mail: atance@posta.unizar.es

†E-mail: cortes@posta.unizar.es 


\section{INTRODUCTION}

Our present understanding of quantum field theory as the low-energy limit of any relativistic quantum-mechanical system [1] has changed our point of view on general problems in quantum field theory such as renormalization [2]. An effective field theory Lagrangian contains an infinite number of terms including the usual ones in a renormalizable Lagrangian (in the power-counting sense [3]) corresponding to the terms with dimension less than or equal to 4 .

A natural way to parametrize the Lagrangian is based on the introduction of a fixed mass scale $M$, which is a characteristic scale of the physical system described by the effective theory, and a dimensionless parameter for each term in the Lagrangian giving the corresponding coefficient in units of $M$ raised to the appropriate power. Ultraviolet divergences can be cancelled by a renormalization of the infinite number of parameters. If one uses an appropriate renormalization scheme (a mass-independent renormalization scheme [4 6]) then, when one computes a process at some energy $E$, the parameter associated to a term in the Lagrangian of dimension $n+4$ gives a contribution proportionalf to $(E / M)^{n}$. If terms suppressed by powers of $(E / M)$ are neglected, the usual renormalizable theory result is recovered, and when one computes to a given order in $(E / M)$ only a finite number of parameters appear. In this sense, although the effective theory has an infinite number of parameters, the theory has predictive power [8]. When the energy becomes comparable to the mass scale $M$ of the effective theory one goes beyond the domain of validity of the effective field theory expansion and one has to consider a new theory, either a new field theory incorporating the appropriate fields to describe the degrees of freedom at these energies, or a theory going beyond the general principles of quantum mechanics and special relativity.

\footnotetext{
*A very clear discussion on this point can be found in [7].
} 
In order to be able to cancel all ultraviolet divergences one usually considers all the terms compatible with some symmetry principles. But this is not necessarily the case. The possibility to have a renormalizable theory with a reduced number of parameters (method of reduction of couplings) has been studied in recent years for different purposes.] The program of reduction of couplings was initiated in [10] by looking for massless renormalizable theories in the power-counting sense with a single dimensionless coupling parameter. The same idea can be applied in the case of effective field theorien looking for relations between the renormalized couplings compatible with the renormalization group equations.

In previous works [12,13] the authors considered the possibility to apply the method of reduction of couplings to the effective field theoretic formulation of quantum gravity. The nonrenormalizability of the theory is not an obstacle to identifying a theory with a finite number of independent parameters. In order to get this result one has to assume that the mass scale associated to the Newtonian limit (Planck mass) is much larger than the mass scale of the effective theory and one has to neglect all the contributions supressed by powers of the ratio of these two mass scales.

The aim of this paper is to apply the same idea to the case of an interaction which does not require us to consider a nonrenormalizable Lagrangian as a starting point. In this case, in contrast to the gravitational interaction, it is not necessary to neglect any contribution in order to identify an effective field theory with a finite number of independent parameters. One can interpret the present work as a step beyond the paradigm of quantum field theory as a low-energy effective theory. Going beyond the renormalizable theory (dominant term in the low-energy limit) does not necessarily imply that we must consider an effective field theory with an infinite number of free parameters. It is possible to consider intermediate

\footnotetext{
${ }^{\dagger}$ For a recent review with a list of references see [9].

$\ddagger$ The idea of considering a reduction of couplings in a non renormalizable theory appears for the first time in [11].
} 
steps. The reduction of couplings, which could be a consequence of a symmetry of the underlying fundamental theory which is hidden in the field theoretical limit, corresponds to a situation where the field theoretical approach goes as far as possible in the sense that the low-energy limit of the theory is only sensitive to the details of the underlying theory through the value of a finite number of parameters. Instead of making reference to a symmetry of a more fundamental theory, a reduction of couplings in an effective theory could be a consequence of the renormalization group flow in the infrared limit with a finite-dimensional infrared fixed manifold. An interpretation along these lines of the reduction of parameters in a renormalizable theory has been considered previously 14 in attempts to determine the values of the Yukawa couplings in the standard model and in the minimal supersymetric standard model.

All the ingredients in the discussion of the application of the method of reduction of couplings in an effective field theory are present in the simplest case, the theory of a real scalar field, which is the subject of this paper. The main result of this work is the identification of a minimal extension of the renormalizable theory of a scalar field. This result is based on a perturbative expansion of the renormalization group equations for the renormalized parameters of the effective theory and therefore one can assume that it is a weakly interacting theory over all the range of validity of the energy expansion.

The triviality of the renormalizable scalar field theory, i.e., the impossibility to describe the interaction of scalar particles over an unlimited range of energies with a $\phi^{4}$ theory, is automatically incorporated when one considers the theory as an effective field theory. As a consequence of the reduction of couplings it is possible to express all the corrections, which are proportional to inverse powers of the mass scale of the effective theory, in terms of a single additional parameter (together with the mass parameter and the quartic self-coupling of the renormalizable $\phi^{4}$ theory). The extension of this result to the standard model of elementary particle physics considered as an effective theory can have interesting physical applications if the characteristic mass scale of the theory is not much larger than the presently available energies. 
In Sec. II we consider the renormalization of the massless scalar field theory considered as an effective field theory. A simple structure for the renormalization group equations is obtained in a mass-independent renormalization scheme due to the absence of a dimensionfull ultraviolet cutoff. The renormalization group equation for the parameter corresponding to a term in the effective Lagrangian of a given dimension does not depend on the parameters corresponding to terms of higher dimension. It is this simple triangular structure which allows us to find a solution to the renormalization group equations, where all the parameters corresponding to terms of dimension greater than 4 in the Lagrangian can be expressed in terms of a single independent parameter $\lambda_{2}$, independently of the value of the renormalization scale. These relations between couplings can be uniquely determined order by order as an expansion in powers of the parameter $\lambda_{0}$ corresponding to the $\phi^{4}$ interaction.

In Sec. III the extension of the reduction of couplings to the massive case is considered. Together with the expansion in powers of the quartic scalar coupling $\lambda_{0}$ one has now an expansion in powers of the mass parameter $\lambda_{-2}$ in the renormalization group equations which gives corrections to the triangular structure of the massless case. The reduction of couplings identified in Sec. II can be extended to this case if one includes an expansion in powers of the product $\lambda_{-2} \lambda_{2}$ in the relations between couplings and one considers the mass parameter $\lambda_{-2}$ as an additional independent parameter.

In Sec. IV the interpretation, limitations and some implications of the effective scalar field theory after reduction of couplings are discussed in detail. The physical content of the reduction of couplings is disentangled from the presence of redundant terms in the effective Lagrangian. A one-to-one correspondence between the three independent parameters of the minimal extension of the renormalized scalar field theory and three mass scales is established. A hierarchy of mass scales in connection with the consistency of the perturbative reduction of couplings and also with the possible ambiguities induced by the high-order behavior of the perturbative expansion (renormalons) is discussed at the level of the effective theory. The standard study of the effective potencial based on the renormalization group is generalized to the case of an effective scalar field theory. The possibility of spontaneous symmetry breaking 
and the modifications required in the discussion of the renormalization and reduction of couplings in the effective field theory are also considered. We end in Sec. V with a summary and prospects.

\section{RENORMALIZATION GROUP EQUATIONS AND REDUCTION OF COUPLINGS: MASSLESS CASE}

The starting point of our discussion is the more general expression for the effective Lagrangian of the theory of a real scalar field invariant under the discrete transformation $\phi \rightarrow-\phi$. It is convenient to introduce a fixed mass scale $M$ as a reference unit for all the couplings of the effective theory. The effective Lagrangian can be written as an expansion in inverse powers of $M$ :

$$
\mathcal{L}_{e f f}=\frac{1}{2} \partial_{\mu} \phi \partial^{\mu} \phi-\frac{\lambda_{0}}{4 !} \phi^{4}+\frac{\vec{\lambda}_{2}}{M^{2}} \overrightarrow{\mathcal{L}}^{(2)}+\frac{\vec{\lambda}_{4}}{M^{4}} \overrightarrow{\mathcal{L}}^{(4)}+\ldots .
$$

A mass $\left(\phi^{2}\right.$ term) has not been included (in next section we will see how the structure of the renormalization group equations is affected in the presence of such a term). The coefficients $\lambda_{0}, \vec{\lambda}_{2}, \vec{\lambda}_{4}, \ldots$, are dimensionless parameters and the power dependence on the mass scale $M$ is fixed by dimensional arguments. The effective field theory expansion has been written in a compact notation where $\overrightarrow{\mathcal{L}}^{(2 n)}$ is a vector whose components are the different terms of dimension $4+2 n$ built out of the scalar field and its derivatives.

For the first terms in the effective field theory expansion one has

$$
\begin{gathered}
\overrightarrow{\mathcal{L}}^{(2)}=\left(\frac{1}{6 !} \phi^{6}, \frac{1}{4} \phi^{2} \partial_{\mu} \phi \partial^{\mu} \phi, \frac{1}{2}(\square \phi)^{2}\right) \\
\overrightarrow{\mathcal{L}}^{(4)}=\left(\frac{1}{8 !} \phi^{8}, \frac{1}{2(4 !)} \phi^{4} \partial_{\mu} \phi \partial^{\mu} \phi, \frac{1}{8}\left(\partial_{\mu} \phi \partial^{\mu} \phi\right)^{2}, \frac{1}{2} \phi \partial_{\mu} \phi \partial^{\mu} \phi \square \phi, \frac{1}{4} \phi^{2}(\square \phi)^{2}, \frac{1}{2} \square \phi \square^{2} \phi\right) .
\end{gathered}
$$

The general parametrization of the effective Lagrangian is redundant for two different reasons. First, a change in the scale $M$ is equivalent to an appropriate rescaling of every dimensionless parameter. A choice of the scale $M$ such that all the dimensionless parameters 
$\lambda_{2 n}^{\left(i_{n}\right)}$ are simultaneously of order 1 allows us to identify $M$ with the scale characteristic of the energy expansion of the effective theory. Second, by using a nonlinear redefinition of fields it is possible to eliminate some of the terms in the effective Lagrangian [15, [16]. Nevertheless it is simpler to use this redundant parametrization in order to identify the general structure of the renormalization group equations.

The standard derivation, in perturbatively renormalizable theories, of the renormalization group equations in a mass-independent renormalization scheme $e^{3}$ can be translated to an effective field theory. An infinite number of counterterms must be admitted in order to absorb the infinities from loop graphs [18. One can prove that limitations on the terms in the bare action arising from symmetries are compatible with renormalizability [19]. The effective theory has an infinite number of bare parameters in one-to-one correspondence with the dimensionless parameters of the effective action. Using dimensional regularization one has expressions for the bare parameters in terms of the renormalized parameters, with poles when $\epsilon \rightarrow 0$ (dimension $D=4-\epsilon$ ). ¿From the independence of the bare parameters on the renormalization scale $\mu$, one concludes that any change of $\mu$ must be equivalent to a change in the renormalized parameters. The renormalization group equations

$$
\mu \frac{d \lambda_{2 n}^{\left(i_{n}\right)}}{d \mu}=\beta_{\lambda_{2 n}^{\left(i_{n}\right)}}(\vec{\lambda})
$$

express this fact. A straightforward generalization of the standard discussion of renormalizable theories [17 leads to a perturbative determination of the renormalization group $\beta$ functions from the residues of the simple poles at $\epsilon=0$ in the relations between bare and renormalized dimensionless parameters.

Dimensional arguments together with the presence of a single mass scale $M$ (the dependence on the renormalization scale $\mu$ is logarithmic) lead to the identification of a simple structure for the renormalization group equations. The $\beta$ functions satisfy the homogeneity conditions

\footnotetext{
§See, for instance, [17].
} 


$$
\beta_{\lambda_{2 n}^{\left(i_{n}\right)}}\left(\vec{\lambda}^{\prime}\right)=t^{2 n} \beta_{\lambda_{2 n}^{\left(i_{n}\right)}}(\vec{\lambda}),
$$

where

$$
\vec{\lambda}_{2 n}^{\prime}=t^{2 n} \vec{\lambda}_{2 n} .
$$

These conditions put strong restrictions on the dependence of the $\beta$ functions on all the dimensionless parameters with one exception, the scalar self coupling $\lambda_{0}$. Each renormalization group $\beta$ function will be a polynomial of a given degree in the parameters $\vec{\lambda}_{2 n}$, $n \neq 0$, with coefficients which are a series expansion in $\lambda_{0}$ determined order by order in perturbation theory.

The explicit form of the renormalization group equations for the first terms in the expansion of the effective Lagrangian is given by

$$
\begin{gathered}
\mu \frac{d \lambda_{0}}{d \mu}=\lambda_{0}^{2} B_{0}, \\
\mu \frac{d \lambda_{2}^{\left(i_{1}\right)}}{d \mu}=\lambda_{0} B_{2}^{\left(i_{1}, j_{1}\right)} \lambda_{2}^{\left(j_{1}\right)}, \\
\mu \frac{d \lambda_{4}^{\left(i_{2}\right)}}{d \mu}=\lambda_{0} B_{4}^{\left(i_{2}, j_{2}\right)} \lambda_{4}^{\left(j_{2}\right)}+B_{4}^{\left(i_{2}, j_{1}, k_{1}\right)} \lambda_{2}^{\left(j_{1}\right)} \lambda_{2}^{\left(k_{1}\right)},
\end{gathered}
$$

where the coefficients $B_{0}, B_{2}$ and $B_{4}$ are power expansions in the self-coupling $\lambda_{0}$. Indices $i_{1}, j_{1}, k_{1}$ take three different values corresponding to the three terms (2.2) in the effective Lagrangian of dimension 6 and $i_{2}, j_{2}$ distinguish the six-dimensionless coefficients of terms of dimension 8 (2.3). A sum over repeated indices $j_{1}, j_{2}, k_{1}$ is understoood in Eqs. (2.8) and (2.9).

The $\mu$ dependence of $\vec{\lambda}_{2 n}$ is fixed by a finite number of parameters $\vec{\lambda}_{2 k}$ with $k \leq n$. This triangular structure of the renormalization group equations allows a systematic search, order by order in the effective theory expansion, of relations between the renormalized parameters independent of the renormalization scale $\mu$ and compatible with the renormalization group equations, i.e., a reduction of couplings. In this way one can consider the possibility to 
have a finite number of independent renormalized parameters despite the appearance of an infinite number of interaction terms in the effective Lagrangian.

The first step in the reduction of couplings is to introduce a dimensionless parameter $\lambda_{2}$ with a renormalization scale dependence given by

$$
\mu \frac{d \lambda_{2}}{d \mu}=\lambda_{0} B_{2} \lambda_{2}
$$

where the coefficient $B_{2}$ is an expansion in powers of $\lambda_{0}$ to be fixed in order to be able to write all the parameters $\lambda_{2}^{\left(i_{1}\right)}$ in terms of $\lambda_{2}$ in a way compatible with the renormalization group equations (2.8). The reduction of couplings at this level corresponds to looking for a relation

$$
\lambda_{2}^{\left(i_{1}\right)}=\ell_{2}^{\left(i_{1}\right)} \lambda_{2}
$$

Consistency with the renormalization group equations leads to

$$
B_{0} \lambda_{0} \frac{d \ell_{2}^{\left(i_{1}\right)}}{d \lambda_{0}}+B_{2} \ell_{2}^{\left(i_{1}\right)}=B_{2}^{\left(i_{1}, j_{1}\right)} \ell_{2}^{\left(j_{1}\right)}
$$

which is a system of equations for the coefficients of the reduction of couplings $\ell_{2}^{\left(i_{1}\right)}$ and the coefficient $B_{2}$ in the $\beta$ function of the independent parameter $\lambda_{2}$. A loop expansion corresponds to a determination in perturbation theory of the $\beta$ functions in Eq. (2.4) and then to a determination of $B_{0}$ and $B_{2}^{\left(i_{1}, j_{1}\right)}$ order by order as an expansion in powers of $\lambda_{0}$ :

$$
\begin{aligned}
B_{0} & =\sum_{k=0}^{\infty} B_{0}^{(k)} \lambda_{0}^{k} \\
B_{2}^{\left(i_{1}, j_{1}\right)} & =\sum_{k=0}^{\infty} B_{2}^{\left(i_{1}, j_{1} ; k\right)} \lambda_{0}^{k} .
\end{aligned}
$$

The perturbative expansion of the renormalization group equations leads to a solution of the consistency equations (2.12) with $\ell_{2}^{\left(i_{1}\right)}$ and $B_{2}$ given as an expansion in powers of $\lambda_{0}$ :

$$
\begin{aligned}
\ell_{2}^{\left(i_{1}\right)} & =\sum_{k=0}^{\infty} \ell_{2}^{\left(i_{1} ; k\right)} \lambda_{0}^{k}, \\
B_{2} & =\sum_{k=0}^{\infty} B_{2}^{(k)} \lambda_{0}^{k},
\end{aligned}
$$


i.e., a perturbative determination of the reduction of couplings. In lowest order, the consistency equation (2.12) reduces to

$$
B_{2}^{(0)} \ell_{2}^{\left(i_{1} ; 0\right)}=B_{2}^{\left(i_{1}, j_{1} ; 0\right)} \ell_{2}^{\left(j_{1} ; 0\right)}
$$

For each eigenvector with a real eigenvalue of the matrix of lowest-order coefficients in the renormalization group equation of $\lambda_{2}^{\left(i_{1}\right)}$ there is a consistent reduction of these parameters. To lowest order in an expansion in powers of $\lambda_{0}$, the coefficients of the independent parameter $\lambda_{2}$ in the reduction equation (2.12) are the components of the eigenvector, and the coefficient of the $\beta$ function of $\lambda_{2}$ is the corresponding eigenvalue. An extension of the reduction of couplings order-by-order in $\lambda_{0}$ leads to an order-by-order approximation of the consistency equation (2.12) which reduces to a linear system of equations for the coefficients $\ell_{2}^{\left(i_{1} ; k\right)}$ of the reduction at each order.

A diagrammatic analysis allows us to identify easily the order in the $\lambda_{0}$ expansion of the first term for each renormalization group coefficient $B_{2}^{\left(i_{1}, j_{1}\right)}$. Just with this information it is possible to identify three different reductions of couplings.

(1) In the first solution the three terms of dimension 6 in the effective Lagrangian have coefficients which begin at the same order in the $\lambda_{0}$ expansion. The coefficients in the reduction of couplings are determined in lowest order, up to an overall normalization factor which can be reabsorbed into a redefinition of the independent parameter $\lambda_{2}$. They are given by

$$
\begin{aligned}
& \ell_{2}^{(1 ; 0)}=1, \\
& \ell_{2}^{(2 ; 0)}=B_{2}^{(2,1 ; 0)} /\left(B_{2}^{(1,1 ; 0)}-B_{2}^{(2,2 ; 0)}\right), \\
& \ell_{2}^{(3 ; 0)}=B_{2}^{(3,2 ; 0)} B_{2}^{(2,1 ; 0)} /\left[B_{2}^{(1,1 ; 0)}\left(B_{2}^{(1,1 ; 0)}-B_{2}^{(2,2 ; 0)}\right)\right] .
\end{aligned}
$$

The extension of the reduction of couplings to all orders is uniquely determined once the arbitrariness in the choice of the independent parameter is used to have $B_{2}=B_{2}^{(1,1 ; 0)}$ for the renormalization group coefficient of the independent parameter $\lambda_{2}$.

(2) A second solution has only terms with derivatives of the field in $\mathcal{L}_{2}$ in lowest order: 


$$
\begin{aligned}
& \ell_{2}^{(1 ; 0)}=0, \\
& \ell_{2}^{(2 ; 0)}=1, \\
& \ell_{2}^{(3 ; 0)}=B_{2}^{(3,2 ; 0)} / B_{2}^{(2,2 ; 0)},
\end{aligned}
$$

and $B_{2}=B_{2}^{(2,2 ; 0)}$.

(3) The last solution has only the term with four derivatives to start with,

$$
\ell_{2}^{(1 ; 0)}=\ell_{2}^{(2 ; 0)}=0, \quad \ell_{2}^{(3 ; 0)}=1
$$

and the $\beta$ function of the independent parameter is proportional to $\lambda_{0}^{2}$ in this case:

$$
B_{2}=\left(B_{2}^{(3,3 ; 1)}-\frac{B_{2}^{(3,2 ; 0)} B_{2}^{(2,3 ; 1)}}{B_{2}^{(2,2 ; 0)}}\right) \lambda_{0} .
$$

Once the reduction of couplings at the level of terms of dimension 6 in the effective Lagrangian has been implemented, the next step is to consider the renormalization group equation for the coefficients of terms of dimension 8. Using the reduction of couplings (2.11) one has

$$
\mu \frac{d \lambda_{4}^{\left(i_{2}\right)}}{d \mu}=\lambda_{0} B_{4}^{\left(i_{2}, j_{2}\right)} \lambda_{4}^{\left(j_{2}\right)}+L_{4}^{\left(i_{2}\right)} \lambda_{2}^{2}
$$

where

$$
L_{4}^{\left(i_{2}\right)}=B_{4}^{\left(i_{2}, j_{1}, k_{1}\right)} \ell_{2}^{\left(j_{1}\right)} \ell_{2}^{\left(k_{1}\right)}
$$

Now one has to look for the possibility to express the parameters $\vec{\lambda}_{4}$ as a function of $\lambda_{0}$ and $\lambda_{2}$ in such a way that one reproduces their renormalization scale dependence, given in Eq. (2.26), as a consequence of the renormalization group equations (2.7), (2.10) of $\lambda_{0}$ and $\lambda_{2}$. A relation

$$
\lambda_{4}^{\left(i_{2}\right)}=\frac{\ell_{4}^{\left(i_{2}\right)}}{\lambda_{0}} \lambda_{2}^{2}
$$

where the coefficient $\ell_{4}^{\left(i_{2}\right)}$ is a function of $\lambda_{0}$, will be consistent with the renormalization group equations if 


$$
B_{0} \lambda_{0} \frac{d \ell_{4}^{\left(i_{2}\right)}}{d \lambda_{0}}+\left(2 B_{2}-B_{0}\right) \ell_{4}^{\left(i_{2}\right)}=B_{4}^{\left(i_{2}, j_{2}\right)} \ell_{4}^{\left(j_{2}\right)}+L_{4}^{\left(i_{2}\right)} .
$$

This is a system of equations for the coefficients in the reduction $\ell_{4}^{\left(i_{2}\right)}$ and all other factors are expansions in powers of $\lambda_{0}$ which are determined order by order either directly from the perturbative approximation to the renormalization group equations or from the perturbative determination of the reduction of couplings at the previous level. A solution of the consistency equations (2.29) with $\ell_{4}^{\left(i_{2}\right)}$ given as an expansion in powers of $\lambda_{0}$,

$$
\ell_{4}^{\left(i_{2}\right)}=\sum_{k=0}^{\infty} \ell_{4}^{\left(i_{2} ; k\right)} \lambda_{0}^{k}
$$

is obtained by solving a linear system of equations at each order in the expansion in powers of $\lambda_{0}$ of the consistency equations.

The steps followed in the determination of the reduction of the parameters corresponding to terms of order $1 / M^{4}$ can be repeated order by order in the expansion in $1 / M$ to get the reduction of the effective scalar field theory. It is given by the relations

$$
\lambda_{2 n}^{\left(i_{n}\right)}=\frac{\ell_{2 n}^{\left(i_{n}\right)}}{\lambda_{0}^{n-1}} \lambda_{2}^{n},
$$

where the coefficients $\ell_{2 n}^{\left(i_{n}\right)}$ are expansions in powers of $\lambda_{0}$,

$$
\ell_{2 n}^{\left(i_{n}\right)}=\sum_{k=0}^{\infty} \ell_{2 n}^{\left(i_{n} ; k\right)} \lambda_{0}^{k}
$$

determined by the renormalization group equations.

The final result is an effective Lagrangian with an infinite number of terms of higher dimension added to the massless renormalizable scalar field Lagrangian but with only one additional independent renormalized parameter $\lambda_{2}$, with a renormalization scale dependence determined by a one loop calculation. In fact we have found three different minimal extensions of the renormalizable theory of this kind.

The reduction of couplings should not be confused with the identification of redundant terms in the effective Lagrangian. By using a nonlinear redefinition of fields it is possible to eliminate all the terms of dimension greater than 4 involving $\square \phi$ [15, [6]. For example, by making the shift of variables $\phi \rightarrow \phi^{\prime}$ with 


$$
\phi=\phi^{\prime}+\left(\frac{\lambda_{2}^{(2)}}{M^{2}}-\frac{1}{2} \lambda_{0} \frac{\lambda_{2}^{(3)}}{M^{2}}\right) \frac{\phi^{\prime 3}}{3 !}-\frac{1}{2} \frac{\lambda_{2}^{(3)}}{M^{2}} \square \phi^{\prime},
$$

one has a Lagrangian with only one term of dimension $6, \hat{\lambda}_{2} / M^{2}(1 / 6 !) \phi^{\prime 6}$, where

$$
\hat{\lambda}_{2}=\lambda_{2}^{(1)}-20 \lambda_{0} \lambda_{2}^{(2)}+10 \lambda_{0}^{2} \lambda_{2}^{(3)}
$$

Then at this level the simplification of the effective Lagrangian due to the presence of redundant terms has a similar effect as the reduction of couplings but this is not the case if one considers higher-dimensional terms. If one includes higher dimensional terms in the change of variables it is possible to extend the simplification of the effective Lagrangian to terms of dimension higher than 6 . At the $1 / M^{4}$ level it is possible to eliminate three out of the six terms in Eq. (2.3) but one still has three new independent parameters to be compared with the absence of any additional free parameters after reduction of couplings.

\section{RENORMALIZATION GROUP EQUATIONS AND REDUCTION OF COUPLINGS: MASSIVE CASE}

If one considers a massive spinless particle then one has to include a term $\lambda_{-2} M^{2} \phi^{2}$ in the Lagrangian density. The dimensionless parameter $\lambda_{-2}$ has to be taken into account in the discussion based on dimensional arguments leading to the general structure of the renormalization group equations. The homogeneity conditions of the $\beta$ functions include the rescaling of the additional parameter $\lambda_{-2}^{\prime}=t^{-2} \lambda_{-2}$ and the simple triangular structure is lost due to the contributions proportional to positive powers of $\lambda_{-2}$ which will be accompanied by parameters corresponding to terms of higher dimensionality. If one wants the reduction of couplings to be applicable also in this case then one has to assume that the dimensionless parameter $\lambda_{-2}$ is sufficiently small to treat its effects as a small perturbation. The reduction of couplings identified in the previous section for the massless case can be taken as the zero-order term of an expansion of the reduction equations in powers of the parameter $\lambda_{-2}$.

The renormalization group equation for the dimensionless parameter associated to the mass term is given by 


$$
\begin{aligned}
\mu \frac{d \lambda_{-2}}{d \mu}= & B_{-2,0} \lambda_{-2} \lambda_{0}+\left[B_{-2,1}^{\left(i_{1}\right)} \lambda_{2}^{\left(i_{1}\right)}\right] \lambda_{-2}{ }^{2}+ \\
& {\left[B_{-2,2}^{\left(i_{2}\right)} \lambda_{4}^{\left(i_{2}\right)}+B_{-2,2}^{\left(i_{1}, j_{1}\right)} \lambda_{2}^{\left(i_{1}\right)} \lambda_{2}^{\left(j_{1}\right)}\right] \lambda_{-2}{ }^{3}+\cdots }
\end{aligned}
$$

where the coeficients $B_{-2, k}$ are power expansions in $\lambda_{0}$ determined from a perturbative calculation of counterterms. As a consequence of the homogeneity conditions, $\beta_{\lambda_{-2}}$ is proportional to $\lambda_{-2}$ and then a vanishing mass parameter considered in Sec. II is consistent with the renormalization group equations. For the self-coupling $\lambda_{0}$ one has

$$
\begin{aligned}
\mu \frac{d \lambda_{0}}{d \mu}= & B_{0,0} \lambda_{0}^{2}+\left[B_{0,1}^{\left(i_{1}\right)} \lambda_{2}^{\left(i_{1}\right)}\right] \lambda_{-2}+ \\
& {\left[B_{0,2}^{\left(i_{2}\right)} \lambda_{4}^{\left(i_{2}\right)}+B_{0,2}^{\left(i_{1}, j_{1}\right)} \lambda_{2}^{\left(i_{1}\right)} \lambda_{2}^{\left(j_{1}\right)}\right] \lambda_{-2}{ }^{2}+\cdots }
\end{aligned}
$$

The first term is just the massless $\beta$ function since $B_{0,0}$ is just the coefficient $B_{0}$ of the massless renormalization group equation. One has additional terms proportional to positive powers of $\lambda_{-2}$ with coefficients $B_{0, k}$ determined perturbatively. The renormalization scale dependence of the parameters corresponding to terms of dimension 6 , which in the massless case was given by Eq. (2.8), will now take the form

$$
\begin{gathered}
\mu \frac{d \lambda_{2}^{\left(i_{1}\right)}}{d \mu}=B_{2,0}^{\left(i_{1}, j_{1}\right)} \lambda_{2}^{\left(j_{1}\right)} \lambda_{0}+\left[B_{2,1}^{\left(i_{1}, i_{2}\right)} \lambda_{4}^{\left(i_{2}\right)}+B_{2,1}^{\left(i_{1}, j_{1}, k_{1}\right)} \lambda_{2}^{\left(j_{1}\right)} \lambda_{2}^{\left(k_{1}\right)}\right] \lambda_{-2}+ \\
{\left[B_{2,2}^{\left(i_{1}, i_{3}\right)} \lambda_{6}^{\left(i_{3}\right)}+B_{2,2}^{\left(i_{1}, j_{1}, i_{2}\right)} \lambda_{2}^{\left(j_{1}\right)} \lambda_{4}^{\left(i_{2}\right)}+B_{2,2}^{\left(i_{1}, j_{1}, k_{1}, \ell_{1}\right)} \lambda_{2}^{\left(j_{1}\right)} \lambda_{2}^{\left(k_{1}\right)} \lambda_{2}^{\left(\ell_{1}\right)}\right] \lambda_{-2}{ }^{2}+\cdots .}
\end{gathered}
$$

Equations (3.1)-(3.3), toghether with its obvious generalization for the remaining parameters in the effective Lagrangian, are the starting point for an extension to the massive case of the reduction of couplings discussed in the previous section. The presence of a new independent parameter $\lambda_{-2}$ and the general structure of the renormalization group equations leads to the consideration in the massive case of a relation

$$
\lambda_{2}^{\left(i_{1}\right)}=\left[\ell_{2,0}^{\left(i_{1}\right)}+\ell_{2,1}^{\left(i_{1}\right)} \frac{\lambda_{2} \lambda_{-2}}{\lambda_{0}^{2}}+\ell_{2,2}^{\left(i_{1}\right)}\left(\frac{\lambda_{2} \lambda_{-2}}{\lambda_{0}^{2}}\right)^{2}+\cdots\right] \lambda_{2},
$$

fixing the effective Lagrangian at order $1 / M^{2}$ in terms of the parameters $\lambda_{-2}, \lambda_{0}$, and $\lambda_{2}$. The coeficients $\ell_{2, k}$ are determined by the consistency of Eq. (3.4) with the renormalization group equations. At each order in the expansion in powers of $\lambda_{-2}$ one has a system of 
equations for the coefficients of the reduction of couplings. In lowest order one has the massless consistency equation (2.12) for $\ell_{2,0}$. At order $\lambda_{-2}$ the consistency of the reduction of $\lambda_{2}^{\left(i_{1}\right)}$ with the renormalization group equations leads to

$$
\begin{array}{r}
\frac{d \ell_{2,0}^{\left(i_{1}\right)}}{d \lambda_{0}} B_{0,1}^{\left(j_{1}\right)} \ell_{2,0}^{\left(j_{1}\right)}+\left(2 B_{2,0}+B_{-2,0}\right) \ell_{2,1}^{\left(i_{1}\right)}+\frac{d \ell_{2,1}^{\left(i_{1}\right)}}{d \lambda_{0}} B_{0,0}= \\
B_{2,0}^{\left(i_{1}, j_{1}\right)} \ell_{2,1}^{\left(j_{1}\right)}+B_{2,1}^{\left(i_{1}, i_{2}\right)} \ell_{4,0}^{\left(i_{2}\right)}+B_{2,1}^{\left(i_{1}, j_{1}, k_{1}\right)} \ell_{2,0}^{\left(j_{1}\right)} \ell_{2,0}^{\left(k_{1}\right)}
\end{array}
$$

which determines the coefficients $\ell_{2,1}^{\left(i_{1}\right)}$ as an expansion in powers of $\lambda_{0}$ once the coefficient $\ell_{4,0}^{\left(i_{2}\right)}$ in the reduction of the parameter $\lambda_{4}^{\left(i_{2}\right)}$ has been determined from the consistency with the renormalization group equations in lowest order [Eq. (2.29)]. This argument can be repeated step by step obtaining an effective Lagrangian with three independent parameters $\lambda_{-2}, \lambda_{0}$, and $\lambda_{2}$. The dimensionless coefficient of a generic term will be given by

$$
\lambda_{2 n}^{\left(i_{n}\right)}=\left[\ell_{2 n, 0}^{\left(i_{n}\right)}+\ell_{2 n, 1}^{\left(i_{n}\right)} \frac{\lambda_{2} \lambda_{-2}}{\lambda_{0}^{2}}+\ell_{2 n, 2}^{\left(i_{n}\right)}\left(\frac{\lambda_{2} \lambda_{-2}}{\lambda_{0}^{2}}\right)^{2}+\cdots\right] \frac{\lambda_{2}^{n}}{\lambda_{0}^{n-1}}
$$

where $\ell_{2 n, k}^{\left(i_{n}\right)}$ are power expansions in $\lambda_{0}$ determined by the consistency with the renormalization group equations. The determination of the reduction coefficients goes from lower to higher values of $k$ (i.e., order by order in the expansion in powers of $\lambda_{-2}$ of the renormalization group equation), for a given value of $k$ it goes from lower to higher values of $n$ (i.e., order by order in the effective Lagrangian expansion), and for a given value of $n$ and $k$ it goes order by order in perturbation theory ( $\lambda_{0}$ expansion). Once a solution for the first coefficients $(n=1, k=0)$ in lowest order has been obtained [Eqs. (2.18)-(2.25)] the reduction of the effective Lagrangian is determined by solving linear systems of equations for the remaining coefficients of the reduction.

The effective theory is defined by the relations giving each coefficient in the effective Lagrangian in terms of the three independent parameters and by the renormalization group equations which give the renormalization scale dependence of the independent parameters. The arbitrariness in the choice of the independent parameter $\lambda_{2}$ has been used in order to have a scale dependence given by Eq. (2.10), where $B_{2}$ is either a constant or a constant times $\lambda_{0}$ depending on the solution to the lowest-order consistency equations. The renormalization 
group equations for $\lambda_{0}$ and $\lambda_{-2}$, which are the parameters of the renormalizable $\phi^{4}$ theory, are given by

$$
\begin{aligned}
\mu \frac{d \lambda_{-2}}{d \mu} & =\left[L_{-2,0}+L_{-2,1} \frac{\lambda_{2} \lambda_{-2}}{\lambda_{0}^{2}}+L_{-2,2}\left(\frac{\lambda_{2} \lambda_{-2}}{\lambda_{0}^{2}}\right)^{2}+\cdots\right] \lambda_{0} \lambda_{-2}, \\
\mu \frac{d \lambda_{0}}{d \mu} & =\left[L_{0,0}+L_{0,1} \frac{\lambda_{2} \lambda_{-2}}{\lambda_{0}^{2}}+L_{0,2}\left(\frac{\lambda_{2} \lambda_{-2}}{\lambda_{0}^{2}}\right)^{2}+\cdots\right] \lambda_{0}^{2},
\end{aligned}
$$

where the coeficients $L_{-2, k}, L_{0, k}$ are obtained by combining the renormalization group equations (3.1), (3.2) with the reduction relations (3.6). One has for the first coefficients

$$
\begin{aligned}
L_{-2,0} & =B_{-2,0} \\
L_{-2,1} & =\lambda_{0} B_{-2,1}^{\left(i_{1}\right)} \ell_{2,0}^{\left(i_{1}\right)} \\
L_{-2,2} & =\lambda_{0} B_{-2,1}^{\left(i_{1}\right)} \ell_{2,1}^{\left(i_{1}\right)}+\lambda_{0}^{2} B_{-2,2}^{\left(i_{2}\right)} \ell_{4,0}^{\left(i_{2}\right)}+\lambda_{0}^{3} B_{-2,2}^{\left(i_{1}, j_{1}\right)} \ell_{2,0}^{\left(i_{1}\right)} \ell_{2,0}^{\left(j_{1}\right)} \\
L_{0,0} & =B_{0,0} \\
L_{0,1} & =B_{0,1}^{\left(i_{1}\right)} \ell_{2,0}^{\left(i_{1}\right)} \\
L_{0,2} & =B_{0,1}^{\left(i_{1}\right)} \ell_{2,1}^{\left(i_{1}\right)}+\lambda_{0} B_{0,2}^{\left(i_{2}\right)} \ell_{4,0}^{\left(i_{2}\right)}+\lambda_{0}^{2} B_{0,2}^{\left(i_{1}, j_{1}\right)} \ell_{2,0}^{\left(i_{1}\right)} \ell_{2,0}^{\left(j_{1}\right)},
\end{aligned}
$$

and then one reproduces the renormalization group equations of the renormalizable $\phi^{4}$ theory plus corrections due to the extension which are determined perturbatively.

\section{SOME ASPECTS OF THE EFFECTIVE SCALAR FIELD THEORY AFTER REDUCTION OF COUPLINGS}

In order to discuss the properties of a scalar field theory considered as a low-energy effective theory with three free parameters it is convenient to introduce a mass scale associated to each of the independent parameters. For the self-coupling $\lambda_{0}$ one can consider the approximation to the renormalization group equation where the corrections proportional to $\lambda_{-2} \lambda_{2}$ are neglected, i.e., the renormalization group equation of the $\phi^{4}$ theory, and at this level one can identify the scale $M_{0}$ at which the perturbative approach breaks down (Landau pole). 
Associated to the parameter $\lambda_{2}$, which controls the departure from the renormalizable $\phi^{4}$ theory, one can consider a new scale $M_{2}$. A comparison of the lowest-order contribution to the $2 \rightarrow 4$ scattering cross section in the renormalizable $\phi^{4}$ theory with the first contribution from the higher-dimensional terms in the effective Lagrangian can be used to define the scale $M_{2}$ as the energy where both contributions become comparable. That leads to the identification of $M_{2}$ as the scale $M$ in the effective Lagrangian (2.1) such that

$$
\hat{\lambda}_{2}\left(\mu=M_{2}\right)=\lambda_{0}^{2}\left(\mu=M_{2}\right)
$$

Note that the scale $M_{2}$ is defined through the parameter $\hat{\lambda}_{2}$ which is the coefficient of the $\phi^{6}$ term in the effective Lagrangian after a shift of variables has been made to eliminate the remaining terms of dimension 6 . Then all the arbitrariness in the parametrization of the effective Lagrangian cancel in the determination of $M_{2}$, as should be since it can be taken as a measure of the energy range where the effective theory expansion is a good aproximation.

The third scale $M_{-2}$ gives a first aproximation to the mass of the spinless particle. It can be introduced by the condition

$$
\lambda_{-2}\left(\mu=M_{-2}\right) M_{2}^{2}=M_{-2}^{2}
$$

on the coefficient $\lambda_{-2}$ of the $\phi^{2}$ term in the effective Lagrangian for $M=M_{2}$. In the determination of the scale $M_{-2}$, as a function of the parameters $\lambda_{-2}$ and $\lambda_{0}$ at the scale $\mu=M_{2}$, the terms proportional to $\lambda_{2}$ in the renormalization group equation of $\lambda_{-2}$ are neglected.

\section{A. Limitations of the minimal extension of the renormalizable $\phi^{4}$ theory}

The renormalizability of the scalar field theory with three independent parameters has been discussed order by order in a perturbative expansion in the self-coupling $\lambda_{0}$. Therefore, unless a generalization of this result at the nonperturbative level is found, one has to assume that $\lambda_{0}(\mu)$ is smaller than the value of the coupling at which perturbation theory becomes 
unreliable for any scale in the range of validity of the effective theory. There are ambiguities in the determination of this value, which in the case of the $\phi^{4}$ theory leads to the identification of $\lambda_{0} \approx 3-4$ as the value at which the theory becomes strongly interacting. ${ }^{* *}$ The conclusion is that the scale $M_{2}$ limiting the range of validity of the effective theory expansion can not exceed the scale $M_{0}$ associated to the parameter $\lambda_{0}$. More precisely one has the condition

$$
\frac{\lambda_{0}\left(\mu=M_{2}\right)}{16 \pi^{2}} \leq \epsilon_{0}
$$

where $\epsilon_{0}$ fixes the domain of validity of the perturbative expansion using several criteria [20] (suppression of higher-order terms, decrease of renormalization scale dependence, absence of significant violations of unitarity,...). A second obvious limitation is that one can only consider low-energy observables such that the ratio $E^{2} / M_{2}^{2}$ is small enough to justify the use of the effective Lagrangian expansion.

The third limitation comes from the expansion in the reduction of couplings due to the introduction of a mass term in the effective Lagrangian. The validity of the step by step reduction of couplings requires that

$$
\frac{\lambda_{2} \lambda_{-2}}{\lambda_{0}^{2}} \leq \epsilon_{2}
$$

over all the energy range of validity of the effective theory. In order to translate this condition into a limitation on the mass scales of the effective theory we have to use the renormalization group equation for the independent parameters and the explicit form of the reduction of couplings.

For definiteness we consider the first reduction, Eqs. (2.18)-(2.20), identified in Sec. II. In this case, neglecting higher-order terms in the $\lambda_{0}$ expansion, one has $\hat{\lambda}_{2}=\lambda_{2}$ and, as a consequence of the definition of the scale $M_{2}, \lambda_{2}\left(M_{2}\right)=\lambda_{0}^{2}\left(M_{2}\right)$. The consistency of the stepby-step reduction leads to the condition $\lambda_{-2}\left(M_{2}\right) \leq \epsilon_{2}$. A one loop calculation determines the lowest-order approximation to the renormalization group equations for the independent parameters which, in the case of the reduction in Eqs. (2.18)-(2.20), reads

\footnotetext{
**For a recent discussion, see 20].
} 


$$
\begin{aligned}
\mu \frac{d}{d \mu}\left(\frac{\lambda_{0}}{16 \pi^{2}}\right) & =3\left(\frac{\lambda_{0}}{16 \pi^{2}}\right)^{2}, \\
\mu \frac{d \lambda_{-2}}{d \mu} & =\lambda_{-2}\left(\frac{\lambda_{0}}{16 \pi^{2}}\right), \\
\mu \frac{d \lambda_{2}}{d \mu} & =15 \lambda_{2}\left(\frac{\lambda_{0}}{16 \pi^{2}}\right) .
\end{aligned}
$$

Taking as a reference the parameters at the scale $M_{2}$, one has a renormalization scale dependence for the self-coupling given by

$$
\lambda_{0}(\mu)=\frac{\lambda_{0}\left(M_{2}\right)}{1+3 / 2\left[\lambda_{0}\left(M_{2}\right) / 16 \pi^{2}\right] \ln \left(M_{2}^{2} / \mu^{2}\right)} .
$$

Then, in this approximation, one has a simple expression for the ratio of scales $M_{0}^{2} / M_{2}^{2}$ in terms of the parameter $\lambda_{0}\left(M_{2}\right)$ :

$$
\frac{M_{0}^{2}}{M_{2}^{2}}=\exp \left[\frac{2}{3} \frac{1}{\lambda_{0}\left(M_{2}\right) / 16 \pi^{2}}\right] .
$$

The solution of the renormalization group equations for the parameters $\lambda_{2}, \lambda_{-2}$ is

$$
\begin{aligned}
\lambda_{-2}(\mu) & =\lambda_{-2}\left(M_{2}\right)\left[\frac{\lambda_{0}(\mu)}{\lambda_{0}\left(M_{2}\right)}\right]^{1 / 3}, \\
\lambda_{2}(\mu) & =\lambda_{2}\left(M_{2}\right)\left[\frac{\lambda_{0}(\mu)}{\lambda_{0}\left(M_{2}\right)}\right]^{5},
\end{aligned}
$$

and then one has, for the combination of parameters which appears in the expansion of the reduction of couplings of the massive case,

$$
\frac{\lambda_{2}(\mu) \lambda_{-2}(\mu)}{\lambda_{0}^{2}(\mu)}=\frac{\lambda_{2}\left(M_{2}\right) \lambda_{-2}\left(M_{2}\right)}{\lambda_{0}^{2}\left(M_{2}\right)}\left[\frac{\lambda_{0}(\mu)}{\lambda_{0}\left(M_{2}\right)}\right]^{10 / 3} .
$$

Since the coupling $\lambda_{0}$ decreases when one goes to lower scales, the convergence of the expansion of the reduction of couplings over all the energy range of validity of the effective theory is automatic once the couplings at the scale $M_{2}$ has been chosen appropriately $\left[\lambda_{-2}\left(M_{2}\right) \leq \epsilon_{2}\right]$.

One can also use the explicit form of the solution of the renormalization group equations to translate the restriction $\lambda_{-2}\left(M_{2}\right) \leq \epsilon_{2}$ into a restriction on the ratio of mass scales $M_{-2}^{2} / M_{2}^{2}$ :

$$
\left[1+\frac{3}{2} \frac{\lambda_{0}\left(M_{2}\right)}{16 \pi^{2}} \ln \left(\frac{M_{2}^{2}}{M_{-2}^{2}}\right)\right]^{1 / 3} \frac{M_{-2}^{2}}{M_{2}^{2}} \leq \epsilon_{2} .
$$


There is a clear correspondence between the limitations on the scales of the effective theory and the different bounds obtained in the $\phi^{4}$ theory. The limitations of perturbation theory in the $\phi^{4}$ theory, including the perturbative unitarity bound, are automatically incorporated in the perturbative approach to the effective Lagrangian and the treatment of the higher-dimensional terms as a small perturbation implies that the perturbative bounds in the effective theory will be very close to the bounds of the $\phi^{4}$ theory.

With respect to the triviality bounds, these are usually formulated as a restriction on the renormalized parameters due to the neccessity of a finite cutoff $\Lambda$ in order to have a nontrivial interacting system. A lattice formulation of the $\phi^{4}$ theory leads [21] to the identification of an upper bound on the scalar mass in units of the cutoff and an upper bound on the renormalized coupling if one limits the size of the deviations from continuum theory. As long as the higher-dimensional terms in the effective Lagrangian are a small perturbation, the result that the bound on the coupling is smaller than the perturbative bound and the conclusion that there is no strongly interacting theory, can be translated to the effective theory justifying its perturbative treatment. It is not clear whether the modification on the perturbative bound for the coupling due to the higher-dimensional terms could be big enough within the domain of validity of the effective theory expansion to make possible a strongly interacting theory.

There is a relation between scaling violations in lattice $\phi^{4}$ theory and deviations of the effective theory from the $\phi^{4}$ theory which can be obtained if one uses the local effective Lagrangian description of scaling violations [22]. An Euclidean lattice $\phi^{4}$ theory with a given lattice action has the same perturbative expansion as an effective scalar theory with a Lagrangian given by the local effective Lagrangian which describes the scaling violations of the lattice theory. The upper bound on the mass in units of the cutoff obtained in the lattice field theory analysis can be translated to the effective scalar theory if one identifies the scale $M_{2}$, which is a measure of the domain of validity of the effective theory expansion, with the cutoff of the lattice $\phi^{4}$ theory. The bounds in the lattice theory on the deviations from the continuum (scaling violations) are associated to the bounds in the effective theory 
on the deviations from the $\phi^{4}$ theory.

Another possible source of limitations of the perturbative treatment of the effective theory is the divergence of the perturbation series. A comparison of the $n$th order term in the $\lambda_{0}$ expansion with the first correction due to higher-dimensional terms leads to the ratio

$$
\frac{\left(\lambda_{0} / 16 \pi^{2}\right)^{n}}{\lambda_{2} \lambda_{-2} / \lambda_{0}^{2}}(\mu)=\frac{\left(\lambda_{0} / 16 \pi^{2}\right)^{n}}{\lambda_{2} \lambda_{-2} / \lambda_{0}^{2}}\left(M_{2}\right)\left[\frac{\lambda_{0}(\mu)}{16 \pi^{2}}\right]^{n-10 / 3}
$$

which, for any given scale $\mu$, becomes smaller than 1 for $n$ sufficiently large. Larger values of the scale require us to go to higher orders in order to have a perturbative correction smaller than the contribution due to higher-dimensional terms. This means that it makes no sense to worry about the large-order behavior of the perturbation series while neglecting the higher-dimensional terms in the effective Lagrangian.

Assuming that the large-order behavior of perturbation theory is dominated by the renormalon singularity [23] leads to an ambiguity in the sum of the perturbation series which is of order $E^{2} / M_{0}^{2}$. Although there is no physical significance to these ambiguities when treated consistently [24] still one can describe the effect of a truncation in the perturbative expansion by these ambiguities [25]. If one has an effective theory with $M_{2}^{2} / M_{0}^{2} \ll 1$, i.e., if the self-coupling at the scale $M_{2}$ is such that $\lambda_{0}\left(M_{2}\right) / 16 \pi^{2} \ll 1$, then the corrections of order $E^{2} / M_{2}^{2}$ due to higher-dimensional terms are much bigger than the ambiguities due to the divergences of the perturbation series. On the contrary if one considers an effective theory where $M_{0}$ and $M_{2}$ are of the same order then the ambiguities due to the divergence in the perturbation series are of the same order as the corrections to the $\phi^{4}$ theory. there is a correspondence between the previous discussion of renormalons in the effective scalar field theory and the connection between scaling violations in lattice $\phi^{4}$ theory and the divergence of the perturbation series [21]. To end this section, let us remark that, although the first of the three possible reductions of couplings of the scalar theory was used in the discussion of the limitations in the effective theory, similar arguments can be used for the

\footnotetext{
${ }^{\dagger \dagger}$ Similar conclusions are obtained from a different point of view in [26].
} 
other cases of reduction of couplings.

\section{B. Physical content of the reduction of couplings}

The cross section for any process in the scalar theory can be written using simple dimensional analysis in the form

$$
\sigma=\frac{1}{E^{2}} \hat{\sigma}\left(x, \lambda_{0}(\mu), \frac{\lambda_{-2}(\mu) M_{2}^{2}}{E^{2}}, \frac{\lambda_{2}(\mu) E^{2}}{M_{2}^{2}}, \frac{\mu^{2}}{E^{2}}\right),
$$

where $E$ is an overall energy scale of the process, $x$ denotes angles and energy ratios and $\mu$ is the renormalization scale. The independence of the cross section $\sigma$ on the renormalization scale can be used to choose $\mu=E$.

In order to apply the effective Lagrangian expansion one has to consider the scattering of jets instead of particles in order to have cross sections which remain finite when the mass vanishes. Using the renormalized parameters corresponding to a mass-independent renormalization scheme, as has been done in the discussion of the renormalization group equations of the effective theory leading to the reduction of couplings, it is possible to expand any cross section in powers of the independent dimensionless parameters. One has

$$
\hat{\sigma}=\sum_{i, j=0}^{\infty} \hat{\sigma}^{(i, j)}\left(\lambda_{0}(E), x\right)\left(\frac{\lambda_{-2}(E) M_{2}^{2}}{E^{2}}\right)^{i}\left(\frac{\lambda_{2}(E) E^{2}}{M_{2}^{2}}\right)^{j},
$$

where the coefficients $\hat{\sigma}^{(i, j)}$ can be determined order by order as an expansion in powers of $\lambda_{0}$ from the perturbative calculation of the cross section and the reduction of couplings. Using

the renormalization group equations for the independent parameters and the definition of the mass scales of the effective theory one can rewrite the cross section in the form

$$
\sigma=\frac{1}{E^{2}} \sum_{i, j=0}^{\infty} \hat{\sigma}^{(i, j)}\left(\lambda_{0}(E), x\right)\left[\frac{\lambda_{-2}(E)}{\lambda_{-2}\left(M_{-2}\right)}\right]^{i}\left[\lambda_{2}(E)\right]^{j}\left(\frac{M_{-2}^{2}}{E^{2}}\right)^{i}\left(\frac{E^{2}}{M_{2}^{2}}\right)^{j},
$$

i.e., as a double expansion in the ratio of the mass of the particle over the energy of the process $M_{-2}^{2} / E^{2}$ and the ratio of the energy of the process over the scale $M_{2}$.

As a consequence of the reduction of couplings in the effective theory it is possible to get a systematic approximation to any cross section in terms of a mass scale $M_{2}$, a selfcoupling $\lambda_{0}\left(M_{2}\right)$, and $\lambda_{-2}\left(M_{2}\right)$ (which fixes the mass of the particle). This is a generalization 
of the result in the $\phi^{4}$ theory which is obtained by taking $\lambda_{2}=0$ in Eq. (4.13). The standard derivation in the $\phi^{4}$ theory of the range of values of the coupling $\lambda_{0}$ for which perturbation theory is reliable [20] can be improved by including the terms with $j \neq 0$ in Eq. (4.13). A measurement of several cross sections, at high enough energy and with a sufficient precision to be sensitive to the corrections due to the higher-dimensional terms, can be used to distinguish the expansion in Eq. (4.13) from the expansion of the most general effective Lagrangian and then to test the validity of the reduction of couplings.

\section{Effective potencial of the effective scalar theory}

Another example of a systematic improvement of the $\phi^{4}$ theory analysis at the level of the effective theory is the application of the renormalization group to the effective potential [27]. The effective potential is the generating functional of one-particle irreducible Green functions evaluated at constant values of the field and its absolute minimum determines the ground state of the theory. It is an effective action (for constant fields) in the sense that it incorporates the effects of loop diagrams but it should not be confused with the action of the effective theory which incorporates the effects of the finite-energy range of validity of the theory. The effective potential of the effective theory incorporates both effects.

The renormalization group equations for the one particle irreducible Green functions of the effective theory yield a renormalization group equation for the effective potential $\mathcal{V}(\phi)$ :

$$
\mu \frac{d \mathcal{V}}{d \mu}+\vec{\beta} \frac{\partial \mathcal{V}}{\partial \vec{\lambda}}=\gamma \phi \frac{\partial \mathcal{V}}{\partial \phi}
$$

which is the generalization of the renormalization group equation for the effective potential of the $\phi^{4}$ theory [27] including all the parameters $\vec{\lambda}$ of the effective theory and their

corresponding $\beta$ functions already introduced in the discussion of the renormalization of the effective theory in Sec. II. Following the standard discussion [27,17] of the renormalization group applied to the effective potential one introduces effective couplings through the equations 


$$
\frac{d \vec{\lambda}}{d t}=\vec{\beta}(\vec{\lambda})
$$

with the initial conditions $\vec{\lambda}(0)=\vec{\lambda}(\mu)$ and the rescaled field:

$$
\bar{\phi}(t)=\exp \left[-\int_{0}^{t} d t^{\prime} \gamma\left(\overrightarrow{\vec{\lambda}}\left(t^{\prime}\right)\right)\right] \phi
$$

Then the renormalization group equation for the effective potential takes the simple form

$$
\frac{d}{d t} \mathcal{V}\left[\bar{\phi}(t), \overrightarrow{\vec{\lambda}}(t), e^{t} \mu\right]=0
$$

which can be trivially solved leading to

$$
\mathcal{V}[\phi, \vec{\lambda}(\mu), \mu]=\mathcal{V}\left[\bar{\phi}(t), \overrightarrow{\vec{\lambda}}(t), e^{t} \mu\right]
$$

Using simple dimensional analysis and obtaining the dependence of the effective potential on the mass scale of the effective theory $M_{2}$, one can use the renormalization group equation to determine the behavior of the effective potential $\mathcal{V}$ as one scales the field $\phi$ :

$$
\mathcal{V}\left[\phi, \vec{\lambda}(\mu), M_{2}, \mu\right]=e^{4 t} \mathcal{V}\left[e^{-t} \bar{\phi}(t), \vec{\lambda}(t), e^{-t} M_{2}, \mu\right]
$$

The large logarithms which appear in a direct perturbative calculation of the potential on the left-hand side dissappear on the right-hand side if one makes the choice $t=\frac{1}{2} \ln \left(\phi^{2} / \mu^{2}\right)$. This assumes that the exponential factor depending on the anomalous dimension in Eq. (4.16) is of order 1. For this choice of t one has

$$
\vec{\lambda}(t)=\vec{\lambda}\left(e^{t} \mu\right)=\vec{\lambda}(\phi)
$$

The validity of the perturbative approach to the effective Lagrangian for $\mu \leq M_{2}$ then justifies a perturbative calculation of the effective potential for $\phi \leq M_{2}$. In lowest order (tree level) one has

$$
\mathcal{V}\left[\phi, \vec{\lambda}(\mu), M_{2}, \mu\right]=\frac{\lambda_{-2}(\phi) M_{2}^{2}}{2} \bar{\phi}^{2}(t)+\frac{\lambda_{0}(\phi)}{4 !} \bar{\phi}^{4}(t)+\frac{\lambda_{2}^{(1)}(\phi)}{6 ! M_{2}^{2}} \bar{\phi}^{6}(t)+\ldots,
$$

i.e., the nonderivative terms in the effective Lagrangian with the dimensionless parameters renormalized at a scale $\mu=\phi$ and the field variable replaced by $\bar{\phi}(t)$. 
The loop expansion for the effective potential in the $\phi^{4}$ theory can be directly translated to the effective theory and then one has a systematic approximation to the effective potential of the effective theory including radiative corrections. The consistency of the approach in Eq. (4.21), where corrections in inverse powers of the mass scale of the effective theory are incorporated before considering loop effects, requires

$$
\frac{\lambda_{0}(\phi)}{16 \pi^{2}} \ll \frac{\bar{\phi}^{2}}{M_{2}^{2}} \approx \frac{\phi^{2}}{M_{2}^{2}} \ll 1 .
$$

This is only possible if $\lambda_{0}\left(M_{2}\right) / 16 \pi^{2} \ll 1$, i.e., if $M_{2}^{2} \ll M_{0}^{2}$, and the approach will apply to the large field behavior of the effective potential.

We end this discussion by pointing out that the consequences in the effective potential of the reduction of couplings at the level of the renormalization of the effective Lagrangian can be trivially identified since the effective potential is determined in terms of the renormalized parameters $\vec{\lambda}(\mu)$ of the effective Lagrangian. This discussion also manifests the difference between the effective Lagrangian (an expansion in powers of the scalar field and derivatives of the scalar field) and the nonanalytic effective potencial.

\section{Spontaneous symmetry breaking and reduction of couplings}

In order to discuss a renormalized effective scalar field theory with spontaneous symmetry breaking it is convenient to start by rephrasing the mechanism of spontaneous symmetry breaking in the $\phi^{4}$ theory at the level of the renormalization group equations. One considers a field $\eta$ which describes the fluctuations around the vacuum of the theory and a Lagrangian

$$
\mathcal{L}^{\prime}(\eta)=\frac{1}{2} \partial_{\mu} \eta \partial^{\mu} \eta-\lambda_{-3}^{\prime} M^{3} \eta-\frac{\lambda_{-2}^{\prime} M^{2}}{2} \eta^{2}-\frac{\lambda_{-1}^{\prime} M}{3 !} \eta^{3}-\frac{\lambda_{0}^{\prime}}{4 !} \eta^{4}+\mathcal{L}_{c . t .}^{\prime}(\eta)
$$

which contains all the terms of dimension less or equal than four without any additional restriction and $\mathcal{L}_{\text {c.t. }}^{\prime}$ denotes the counterterms required to renormalize the theory. The coef-

ficient of the linear term $\lambda_{-3}^{\prime}$ is fixed by the condition that the vacuum expectation value of $\eta$ is zero (in lowest order this leads to $\lambda_{-3}^{\prime}=0$ but this is not so in higher orders). 
A one loop calculation leads to the renormalization group equations

$$
\begin{gathered}
\mu \frac{d \lambda_{-3}^{\prime}}{d \mu}=-\lambda_{-1}^{\prime} \lambda_{-2}^{\prime}, \\
\mu \frac{d \lambda_{-2}^{\prime}}{d \mu}=\lambda_{-1}^{\prime 2}-\lambda_{0}^{\prime} \lambda_{-2}^{\prime}, \\
\mu \frac{d \lambda_{-1}^{\prime}}{d \mu}=3 \lambda_{0}^{\prime} \lambda_{-1}^{\prime}, \\
\mu \frac{d \lambda_{0}^{\prime}}{d \mu}=3 \lambda_{0}^{\prime 2},
\end{gathered}
$$

where an overall coefficient $1 / 16 \pi^{2}$ has been reabsorbed into a rescaling of all the dimensionless parameters.

The case of spontaneous symmetry breaking corresponds to the possibility to find a translation of the variable $\eta \rightarrow \phi=\eta+v$ such that when the Lagrangian is written in terms of the variable $\phi$ only terms invariant under the transformation $\phi \rightarrow-\phi$ appear,

$$
\mathcal{L}(\phi)=\mathcal{L}^{\prime}(\phi-v)=\frac{1}{2} \partial_{\mu} \phi \partial^{\mu} \phi-\frac{\lambda_{-2} M^{2}}{2} \phi^{2}-\frac{\lambda_{0}}{4 !} \phi^{4}+\mathcal{L}_{\text {c.t. }}(\phi) .
$$

The effect of a general translation of the field variable $\phi \rightarrow \phi+M v$ is equivalent to a change of dimensionless parameters:

$$
\begin{gathered}
\lambda_{-3}^{\prime}=\lambda_{-3}+v \lambda_{-2}+\frac{1}{2} v^{2} \lambda_{-1}+\frac{1}{3 !} v^{3} \lambda_{0}, \\
\lambda_{-2}^{\prime}=\lambda_{-2}+v \lambda_{-1}+\frac{1}{2} v^{2} \lambda_{0}, \\
\lambda_{-1}^{\prime}=\lambda_{-1}+v \lambda_{0}, \\
\lambda_{0}^{\prime}=\lambda_{0} .
\end{gathered}
$$

If one combines these relations, valid for an arbitrary translation of the field variables, with the equation determining $\lambda_{-3}^{\prime}$ in terms of the remaining parameters in $\mathcal{L}^{\prime}\left(\lambda_{-3}^{\prime}=0\right.$ in 
lowest order) and uses the symmetry of $\mathcal{L}$ in the case of spontaneous symmetry breaking $\left(\lambda_{-3}=\lambda_{-1}=0\right)$ then one can determine the vacuum expectation value $v$ of the field $\phi$ in terms of the parameters $\lambda_{-2}$ and $\lambda_{0}$. When the renormalization group equations (4.24)(4.27) are rewritten in terms of the parameters $\lambda_{k}$ corresponding to this value of $v$ then one finds

$$
\mu \frac{d \lambda_{-3}}{d \mu}=\mu \frac{d \lambda_{-1}}{d \mu}=0,
$$

which is the manifestation at this level of the renormalizability of the $\phi^{4}$ theory with spontaneous symmetry breaking, and the renormalization group equations for the two parameters of the theory become

$$
\begin{aligned}
\mu \frac{d \lambda_{-2}}{d \mu} & =5 \lambda_{0} \lambda_{-2} \\
\mu \frac{d \lambda_{0}}{d \mu} & =3 \lambda_{0}^{2} .
\end{aligned}
$$

Once the spontaneous breaking of the symmetry has been formulated at the level of the renormalization group equations for the $\phi^{4}$ theory in a mass-independent renormalization scheme the generalization to the case of the effective scalar theory is trivial. Instead of the Lagrangian $\mathcal{L}^{\prime}$ in Eq. (4.23) one has to consider all possible terms with any dimension and instead of the renormalization group equations in Eqs. (4.24)-(4.27) one now has the renormalization group equations for the parameters of the effective scalar theory, including the parameters $\vec{\lambda}_{2 n+1}^{\prime}$ associated to terms with an odd number of fields. The structure of the renormalization group equations, based on dimensional arguments and the expansion in powers of $\lambda_{0}$, is not modified by the addition of odd terms. Once more a translation $v$ of the field variable is determined such that the effective Lagrangian in the translated field variable is invariant under $\phi \rightarrow-\phi$. Its value is determined as a double expansion in powers of $\lambda_{0}$ and $\lambda_{-2}$ from the condition that the vacuum expectation value of the field $\eta$ vanishes.

When the renormalization group equations for the parameters $\vec{\lambda}^{\prime}$ of the effective theory are written in terms of the parameters of the Lagrangian which results from a translation 
of the field variable by the value of $v$ determined previously then one finds once more Eq. (4.33) and

$$
\mu \frac{d \vec{\lambda}_{2 n+1}}{d \mu}=0
$$

which is the manifestation at this level of the renormalizability of the effective theory with spontaneous symmetry breaking. One also has renormalization group equations for the parameters $\lambda_{-2}, \lambda_{0}, \lambda_{2 n}^{i_{n}}$ which are the analogue for the effective scalar theory with spontaneous symmetry breaking of the renormalization group equations of the effective theory in the symmetric case discussed in Sec. III.

Since the structure of the renormalization group equation for $\lambda_{2 n}^{i_{n}}$ is fixed by dimensional arguments which are not affected by the spontaneous breaking of the symmetry, the step-bystep determination of a reduction couplings applies also in this case. In fact the lowest-order term in the expansion in powers of $\lambda_{-2}$ (zero-order term), which is the term identifying the possible reductions of couplings, is the one found in the symmetric case. Then one has a one-to-one correspondence between the reduction of couplings in the symmetric and spontaneously broken cases and the differences appear in the extension of the reduction to higher orders in the expansion in powers of $\lambda_{-2}$.

The conclusion of this discussion is that there is no obstruction to translating all the analysis of the symmetric effective theory to the case of spontaneous symmetry breaking. This means that all the bounds found in the $\phi^{4}$ theory 28 with spontaneous symmetry breaking can be discussed also at the level of the effective theory as we argued before in the symmetric case. In particular the stability bounds on the theory derived from the large field behavior of the effective potential for a scalar field coupled to fermionic fields [29] can be an example where the higher-dimensional terms in the effective theory can be important depending on the ratio of scales of the effective theory. 


\section{SUMMARY AND OUTLOOK}

It has been shown that the perturbative renormalization of the theory of a scalar field with nonrenormalizable couplings (negative dimension) which requires an infinite number of counterterms, is compatible with the presence of only a finite number of independent parameters. The renormalization group equations of the effective theory allow us to identify three possible extensions of the $\phi^{4}$ theory with an additional dimensionless parameter $\lambda_{2}$ which controls the contribution of all the higher-dimensional terms. The extension is determined order by order in a double expansion in the quartic coupling $\lambda_{0}$ and the product $\lambda_{-2} \lambda_{2}$, where $\lambda_{-2}$ is the dimensionless parameter associated to the mass term.

Assuming the validity of perturbation theory and of the effective theory energy expansion, it is possible to improve systematically the bounds on the $\phi^{4}$ theory due to triviality both in the symmetric and the spontaneously broken cases. If one assumes that the fundamental theory at higher energies is such that its low-energy limit is described by one of the minimal extensions of the $\phi^{4}$ theory then the modifications to the bounds due to the higher dimensional terms can be calculated in terms of just one additional parameter with respect

to the renormalizable $\phi^{4}$ theory. An analysis along these lines of a minimal extension of the standard model could have important physical implications if the characteristic mass scale of this effective theory is not much higher than the Fermi scale. The first step in this direction would be to generalize the discussion of the effective scalar field theory including a set of fermionic fields coupled to a set of scalar fields corresponding to the standard model neglecting the gauge interactions.

Another possible extension of the present discussion of the method of reduction of couplings is to consider the possibility of a ressumation of terms in the double expansion which goes beyond the step-by-step reduction of couplings considered in this work. The possibility to have a fixed point of the renormalization group equations of an effective theory not corresponding to a free theory and having a finite-dimensional domain of attraction (asymptotic safety 30]) can be seen as an example of a reduction of couplings. The restriction to the 
finite-dimensional surface, consisting of the trajectories of the renormalization group which are attracted into the fixed point, defines a set of infinite relations among couplings compatible with renormalizability leading to a theory with a finite number of free parameters.

The interpretation of a lattice $\phi^{4}$ theory as an effective scalar field theory, based on the local effective Lagrangian which reproduces the asymptotic small lattice spacing expansion of Green functions, provides a new framework with which to discuss the reduction of couplings. In fact the improvement program [22] can be interpreted as the identification of suitable irrelevant terms to be added to the lattice action leading to an effective scalar theory whose first correction to the renormalizable $\phi^{4}$ theory has higher and higher dimension as one goes to higher and higher orders in the improvement. The final result of the improvement program is a lattice field theory which is an effective field theory with a trivial reduction of couplings (all the parameters corresponding to irrelevant terms in the effective Lagrangian vanish). Each step in the improvement can be seen as a reduction of couplings of a different type of the reduction of couplings considered in this work. The improvement at order $\mathrm{k}$ corresponds to $\lambda_{2 n}^{i_{n}}=0,0<n<k$, which is another way to fix arbitrary parameters in the effective Lagrangian in a way compatible with the renormalization group equations. On the other hand, the reduction of couplings we have studied corresponds to a different way to fix arbitrary parameters; one first looks for renormalization group invariant relations among the parameters $\lambda_{2}^{i_{1}}$, next one finds expressions for $\lambda_{4}^{i_{2}}$ in terms of the independent parameter of the previous step, and one extends the procedure order by order in the energy expansion of the effective Lagrangian.

The identification of a lattice action, with irrelevant terms fixed in such a way that its local effective Lagrangian coincides with the effective Lagrangian of one of the minimal extensions of the renormalizable $\phi^{4}$ theory, suggests the possibility of a reformulation of the reduction of couplings at the level of lattice field theory. This could allow the discussion of the method of reduction of couplings at the nonperturbative level. Finally, it would be interesting to find an example of the realization of the idea of reduction of couplings in a simple enough theory to be able to determine its low-energy limit as a minimal extension of 
a renormalizable theory.

\section{Acknowledgments}

This work was partially supported by CICYT contract AEN 96-1670. The work of M.A. has been supported by DGA. 


\section{REFERENCES}

[1] S. Weinberg, The Quantum Theory of Fields (Cambridge University Press, Cambridge, England, 1995)

[2] J. Polchinski, Nucl. Phys. B231, 269 (1984).

[3] F. J. Dyson, Phys. Rev. 75, 486 (1949); 75, 1736 (1949).

[4] S. Weinberg, Phys. Rev. D8, 3497 (1973).

[5] E. Brezin, J. C. Le Gillou and J. Zinn-Justin, in Field Theoretical approach to critical phenomena, Phase transitions and critical phenomena Vol. 6, edited by C. Domb and M. S. Green (Academic Press, London, 1976)

[6] G. 't Hooft, Nucl. Phys. B61, 455 (1973).

[7] A. V. Manohar, hep-ph/9508245.

[8] S. Weinberg, Physica 96A, 327 (1979); H. Georgi, Weak Interactions and Modern Particle Theory, (Benjamin Cummings, Menlo Park, CA, 1984); J. Gasser and H. Leutwyler, Nucl. Phys. B250, 465 (1985); J. F. Donoghue, E. Golowich and B. R. Holstein, Dynamics of the Standard Model (Cambridge University Press, Cambridge, England 1992).

[9] R. Oehme, hep-th/9511006.

[10] R. Oehme and W. Zimmerman, Commun. Math. Phys. 97, 569 (1985); W. Zimmerman, ibid. 97, 211 (1985).

[11] L. A. W. Toro, Z. Phys. C56, 635 (1992).

[12] M. Atance and J. L. Cortés, Phys. Lett. B387, 697 (1996).

[13] M. Atance and J. L. Cortés, Phys. Rev. D54, 4973 (1996).

[14] B. Schrempp and M. Wimmer, hep-ph/9606386. 
[15] H. Georgi, Nucl. Phys. B361, 339 (1991).

[16] C. Arzt, Phys. Lett. B342, 189 (1995).

[17] D. J. Gross, in Methods in Field Theory, Proceedings of the Les Houches Summer School, Les Houches, France, 1975, edited by R. Balian and J. Zinn-Justin, Les Houches Summer School Proceedings Vol. XXVIII (North Holland, Amsterdam, 1976).

[18] W. Zimmermann, in Lectures on Elementary Particles and Quantum Field Theory, 1970 Brandeis University Summer Institute in Theoretical Physics Vol. 1, edited by S. Deser, M. Grisaru and H. Pendleton, (M.I.T. Press, Cambridge, Massachusetts, 1970).

[19] J. Gomis and S. Weinberg, Nucl. Phys. B469, 473 (1996).

[20] K. Riesselmann ans S. Willenbrock, Phys. Rev. D55, 311 (1997).

[21] M. Lüscher and P. Weisz, Nucl. Phys. B290, 25 (1987).

[22] K. Symanzik, Nucl. Phys. B226, 187 (1983).

[23] B. Lautrup, Phys. Lett. B69, 109 (1977); G. 't Hooft, in The Whys of Subnuclear Physics (Erice 1977), edited by A. Zichichi (Plenum, New York, 1979); G. Parisi, Phys. Lett. B76, 65 (1978); G. Parisi, Phys. Rep. 49, 215 (1979).

[24] M. Luke, A. V. Manohar and M. J. Savage, Phys. Rev. D51, 4924 (1995).

[25] G. Martinelli and C. T. Sachrajda, Nucl. Phys. B478, 660 (1996).

[26] D. Espriu and R. Tarrach, Phys. Lett. B383, 482 (1996).

[27] S. Coleman and E. Weinberg, Phys. Rev. D7, 1888 (1973).

[28] R. Dashen and H. Neuberger, Phys. Rev. Lett. 50, 1897 (1983); M. Lüscher and P. Weisz, Nucl. Phys. B295, 65 (1988).

[29] N. Cabibbo, L. Maiani, G. Parisi and R. Petronzio, Nucl.Phys. B158, 295 (1979). 
[30] S. Weinberg, in General Relativity, edited by S. W. Hawking and W. Israel, (Cambridge University Press, Cambridge, England, 1979). 